\title{
Active Release of Human Platelet Factor VIII-Related Antigen by Adenosine Diphosphate, Collagen, and Thrombin
}

\author{
J. Koutts, P. N. Walsh, E. F. Plow, J. W. Fenton, II, B. N. Bouma, and \\ T. S. Zimmerman, Department of Molecular Immunology, Scripps Clinic and \\ Research Foundation, La Jolla, California 92037
}

\begin{abstract}
A B S T R A C T Platelet Factor VIII-related antigen (VIIIR:Ag) represents a significant proportion of the total circulating VIIIR:Ag pool. However, its participation in the events of primary hemostasis has not been shown. We now report that platelet-contained VIIIR:Ag is released from platelets by collagen, ADP and thrombin. The concentrations of these agonists, required for VIIIR:Ag release, are the same or lower than those required for release of serotonin, lysosomal enzymes, or fibrinogen. This release has the features of an energydependent secretory response because it is blocked by the metabolic inhibitors, antimycin $A$ and 2-deoxyD-glucose. The electrophoretic characteristics of the VIIIR:Ag released by collagen and ADP are similar to those of plasma VIIIR:Ag. However, thrombin-released platelet VIIIR:Ag differs from that of plasma in that the less anodal forms are relatively depleted. These differences do not appear to be the result of proteolytic degradation of platelet-derived VIIIR:Ag, but may reflect interactions between specific molecular forms of VIIIR:Ag and the platelet membrane. These studies sug-
\end{abstract}

This work has been presented in part at The American Federation for Clinical Research National Meeting (San Francisco, 30 April-2 May 1978), and published in abstract form. 1978. Clin. Res. 26: 350A.

Dr. Walsh is a recipient of a National Institute of Health Research Career Development Award (HL-70545). Dr. Koutts' present address is Department of Medicine, Monash University Medical School, Alfred Hospital, Prahran 3181, Victoria, Australia; Dr. Walsh's, Department of Medicine, Temple University Health Science Center, Philadelphia, Pa. 19140; Dr. Plow, Scripps Clinic and Research Foundation, La Jolla, Calif. 92037; Dr. Fenton's, Division of Laboratories and Research, New York State Department of Health, Albany, N. Y. 12201; Dr. Bouma's, University Hospital Department of Internal Medicine, Catharijnesingel 101, Utrecht, The Netherlands.

Received for publication 1 May 1978 and in revised form 21 July 1978. gest mechanisms by which platelet-contained VIIIR:Ag may contribute to the primary events of hemostasis.

\section{INTRODUCTION}

Factor VIII-related antigen (VIIIR:Ag) ${ }^{1}$ is a protein that appears to be necessary for normal platelet function. In von Willebrand's disease, this protein is either markedly decreased in the plasma (1) or is qualitatively abnormal (2). These defects are associated with a prolonged bleeding time and decreased ristocetin-induced platelet aggregation (3). Experimentally, VIIIR:Ag deficiency has been correlated with a decreased adhesion of platelets to subendothelium (4). In plasma, VIIIR:Ag is also associated with the Factor VIII procoagulant activity (VIIIR:C), although the precise relationship between the two remains unclear. VIIIR:Ag has also been demonstrated in platelets (5-10), megakaryocytes (11, $12)$, and the endothelial cell $(11,13)$.

The platelet contains $10-15 \%$ of the total circulating VIIIR:Ag $(5,6)$. Although VIIIR:Ag is synthesized in both the endothelial cell (14) and the megakaryocyte (15), little is known about the physiology of cellular VIIIR:Ag, and no functional role has been demonstrated for it.

We report here that a significant proportion of platelet VIIIR:Ag is released by stimulation with three physiologic agonists: ADP, collagen, and thrombin. This release has the features of a true secretory process because it is prevented by agents that inhibit energydependent functions by depleting the platelet metabolic pool of ATP. The kinetic features of VIIIR:Ag secretion by platelets in response to graded doses of collagen

\footnotetext{
${ }^{1}$ Abbreviations used in this paper: LDH, lactic dehydrogenase; 5HT, 5-hydroxytryptamine (serotonin); VIIIR:Ag, Factor VIII-related antigen; VIIIR:C, Factor VIII procoagulant activity; VIIIR:RCo, platelet-ristocetin cofactor activity.
} 
or thrombin are distinct from those of 5-hydroxytryptamine (5HT) (a dense body marker) or lysosomal enzyme ( $\alpha$-granule marker) secretion. The maximum amount of VIIIR:Ag releasable by thrombin is significantly less than is releasable by collagen. Moreover, the electrophoretic characteristics of VIIIR:Ag released into the supernate by platelets in response to ADP or collagen are different from those of VIIIR:Ag released in response to thrombin.

\section{METHODS}

Preparation of washed platelets. $9 \mathrm{vol}$ of blood were collected by aseptic venipuncture with a 16-gauge needle and catheter into $1 \mathrm{vol}$ of $3.8 \% \mathrm{wt} / \mathrm{vol}$ trisodium citrate using plastic equipment and containers throughout. Platelet-rich plasma was obtained by centrifugation at $300 \mathrm{~g}$ for $15 \mathrm{~min}$. Plateletrich plasma was incubated with $0.5 \mu \mathrm{Ci}\left[2{ }^{14} \mathrm{C}\right] 5 \mathrm{HT}$ binoxolate (New England Nuclear, Boston, Mass.) per $20 \mathrm{ml}$ for $40 \mathrm{~min}$ at $37^{\circ} \mathrm{C}$. Platelets were then washed twice by albumin density gratient separation at ph 6.5 in a calcium-free Tyrode's solution composed of: $\mathrm{NaCl}(8.0 \mathrm{~g} /$ /iter $), \mathrm{KCl}(0.2 \mathrm{~g} /$ liter $), \mathrm{NaH}_{2} \mathrm{PO}_{4} \cdot 2 \mathrm{H}_{2} \mathrm{O}$ (0.065 g/liter), $\mathrm{MgCl}_{2} \cdot 6 \mathrm{H}_{2} \mathrm{O}$ (0.415 g/liter), $\mathrm{NaHCO}_{3}(1.0 \mathrm{~g} /$ liter), dextrose ( $1.0 \mathrm{~g} /$ liter $)$, and bovine serum albumin (1.0 g/liter; Sigma Chemical Co., St. Louis, Mo.) as described (16) with the exception that apyrase was not used. Platelets for ADP stimulation were taken directly from the second albumin gradient and diluted with $3 \mathrm{vol}$ of calcium-free Tyrode's solution ( $\mathrm{pH}$ 7.4) and $1 \mathrm{vol}$ of citrated plasma obtained from a patient with severe (homozygous) von Willebrand's disease which contained $<0.0005$ U VIIIR: $\mathrm{Ag} / \mathrm{ml}$. For all other studies, after albumin density gradient washing, the platelets were gel filtered on a $25 \times 1.6-\mathrm{cm}$ column of Sepharose 2B (Pharmacia Fine Chemicals, Uppsala, Sweden) in the calcium-free Tyrode's solution at $\mathrm{pH}$ 7.4. Platelets were counted electronically with a model Z particle counter (Coulter Electronics, Inc., Hialeah, Fla.). This technique gave final platelet counts between $5.5 \times 10^{8}$ and $8.0 \times 10^{8}$ platelets $/ \mathrm{ml}$.

Experiments with washed platelets. Imipramine (CibaGeigy Corp., Pharmaceuticals Div., Summit, N. J.) was addea to washed platelet suspensions at a final concentration of 5 $\mu \mathrm{M}$. This concentration has been determined to inhibit over $90 \%$ of the uptake of $5 \mathrm{HT}$ by platelets. Where metabolic inhibitor studies were performed, the washed platelets were incubated with a final concentration of $4 \mu \mathrm{g} / \mathrm{ml}$ antimycin $\mathrm{A}$ (Calbiochem, La Jolla, Calif.) and $6 \mathrm{mM}$ 2-deoxy-D-glucose (Sigma Chemical Co.) for $30 \mathrm{~min}$ at $37^{\circ} \mathrm{C}$ (17). Stock solutions were antimycin $\mathrm{A}, 2 \mathrm{mg} / \mathrm{ml}$ in ethanol and stored at $-70^{\circ} \mathrm{C}$ and 2-deoxy-D-glucose, $600 \mathrm{mM}$ in $50 \mathrm{mM}$ isotonic imidizole buffer, ph 7.3 , and stored at $-20^{\circ} \mathrm{C}$. Aggregating agents used were: ADP (grade I; Sigma Chemical Co.) from a stock solution of $1 \mathrm{mM}$ ADP in calcium-free Tyrode's solution, $\mathrm{pH}$ 7.4, human adult skin collagen (a gift from Dr. G. Wilner, Jewish Hospital, St. Louis, Mo.) prepared by the Nishihara method $(18,19)$ from a stock solution of $5 \mathrm{mg} / \mathrm{ml}$ in $0.01 \mathrm{M}$ Tris, $0.14 \mathrm{M}$

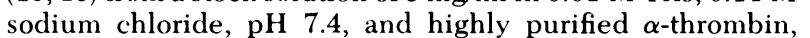
prepared and handled as described (20), in a stock solution of $9,000 \mathrm{IU} / \mathrm{ml}$ in $0.75 \mathrm{M} \mathrm{NaCl}$ and kept at $-70^{\circ} \mathrm{C}$. All dilutions of these reagents were made with calcium-free Tyrode's solution, $\mathrm{pH}$ 7.4. Aggregation was performed by adding a $10 \% \mathrm{vol}$ aliquot of the aggregating agent to a continuously stirred (750 $\mathrm{rpm})$ suspension of platelets in siliconized aggregometer cuvettes at $37^{\circ} \mathrm{C}$. The stirring was continued for exactly $90 \mathrm{~s}$ in all cases and then the platelets were allowed to stand at $37^{\circ} \mathrm{C}$. For the dose-response studies, the period of reaction was terminated by centrifugation at $7,000 \mathrm{~g}$ for $2 \mathrm{~min}$. For the ma- jority of time-course studies, the reactions were terminated by the addition of $5 \%$ by volume of a solution of formalin $(0.4 \mathrm{M})$ and EDTA $(0.06 \mathrm{M})$ giving final concentrations of 20 $\mathrm{mM}$ formalin and $3 \mathrm{mM}$ EDTA (21). Matched studies determined that the addition of formalin-EDTA did not significantly affect the assay of VIIIR:Ag, 5HT, $N$-acetyl- $\beta$-Dglucosamidase or $\beta$-glucuronidase, but did affect the measurement of fibrinogen. For this reason, time-course studies involving fibrinogen release were terminated by centrifugation only. Time points of $<5 \mathrm{~min}$ are therefore not given in these studies. In all studies, aggregation cuvettes were centrifuged at $7,000 \mathrm{~g}$ for $2 \mathrm{~min}$ at room temperature, the supernate was removed and then recentrifuged at $7,000 \mathrm{~g}$ for an additional 10 $\min$ at $4^{\circ} \mathrm{C}$. The supernate was then collected and immediately stored at $4^{\circ} \mathrm{C}$. Platelets were lysed either by freeze-thawing in liquid nitrogen four times, or by the addition of a $10 \% \mathrm{vol}$ of a $10 \%$ solution of Triton X-100 (J. T. Baker Chemical Co., Phillipsburg, N. J.).

Dose-response studies. Collagen was used in final concentrations of $1,5,10,20,50,125$, and $250 \mu \mathrm{g} / \mathrm{ml}$. Thrombin was used in final concentrations of $0.01,0.025,0.05,0.1,0.25,0.5$, 1.0 , and $10 \mathrm{U} / \mathrm{ml}$. Five studies were performed with each concentration of each agent in which the reaction was stopped 10 min after the addition of incubating agents and two studies after $60 \mathrm{~min}$ of incubation. No VIII:Ag was released at the end of $60 \mathrm{~min}$ if no agonists were added. ADP was studied at $0.3 \mu \mathrm{M}, 1 \mu \mathrm{M}, 3 \mu \mathrm{M}, 10 \mu \mathrm{M}, 50 \mu \mathrm{M}$ and $0.1 \mathrm{mM}$ final concentrations. Five studies were performed with each concentration of ADP, all of 60 min duration.

A number of studies were performed to determine if thrombin, or a substance released from platelets by thrombin, affected VIIIR:Ag, either quantitatively or qualitatively. Thrombin $(0.5 \mathrm{U} / \mathrm{ml}$, final concentration) was added to the supernate of platelets $1 \mathrm{~h}$ after their stimulation by collagen ( $50 \mu \mathrm{g} / \mathrm{ml}$, final concentration). Alternatively, highly purified VIIIR: $\mathrm{Ag}(0.04 \mathrm{U} / \mathrm{ml}$, final concentration) of relatively slow electrophoretic mobility obtained from an early void volume fraction (22) was added to the supernate of platelets obtained 3 and $60 \mathrm{~min}$ after stimulation with thrombin or after freezethaw lysis. After incubation for an additional hour, the VIIIR:Ag of each sample was compared with the untreated material for any evidence of alteration in amount or electrophoretic mobility.

Time-course studies. Reactions were stopped by the addition of formalin EDTA at $10,30,60,120 \mathrm{~s}, 5,20,45$, and $60 \mathrm{~min}$. The zero-time control for these experiments was a platelet suspension to which the appropriate stimulus was added $10 \mathrm{~s}$ after the formalin-EDTA and allowed to incubate for $60 \mathrm{~min}$. Thrombin was used in a final concentration of $0.5 \mathrm{U} / \mathrm{ml}$, collagen at $50 \mu \mathrm{g} / \mathrm{ml}$, and ADP at $50 \mu \mathrm{M}$. Five time-course studies were performed with each agent. In three of these studies, metabolically inhibited platelets were studied concurrently.

Radio-crossed immunoelectrophoresis and radio-quantitated immunoelectrophoresis. These techniques were performed as described previously $(23,24)$ with the exception that ${ }^{125}$ affinity purified rabbit anti-VIIIR:Ag was mixed with unlabeled "native" rabbit anti-VIIIR:Ag and immunoprecipitates were detected with autoradiography instead of Coomassie Blue staining. This allowed the use of more dilute antibody and hence increased the sensitivity of these techniques. (As little as $0.0005 \mathrm{U}$ of VIIIR:Ag could be detected.) Affinity purification of the rabbit anti-VIIIR:Ag was carried out as described elsewhere $(25,26)$. An immunoglobulin (Ig)G fraction of high titer monospecific anti-VIIIR:Ag antisera (22) was prepared by ammonium sulfate fractionation and DEAE chromatography. This IgG was then applied to partially purified VIIIR:Ag (250 $\mu \mathrm{g}, 25 \mathrm{U})$ which had been cross-linked 
to $1 \mathrm{ml}$ agarose by gluteraldehyde $(25,26)$. The partially purified VIIIR:Ag was obtained by agarose gel chromatography of a commercial Factor VIII preparation. $250 \mu \mathrm{g}$ of rabbit antibody bound to these beads and $100 \mu \mathrm{g}$ could be subsequently eluted at pH 2.2. This affinity purified antibody was labeled by the chloramine $\mathrm{T}$ technique to a sp act $=5 \mu \mathrm{Ci}$ ${ }^{125} \mathrm{I} / \mu \mathrm{g}(27)$. The affinity purified antibody was monospecific detecting only VIIIR:Ag as analyzed in crossed immunoelectrophoresis of plasma or platelet protein (Figs. 5 and 6) or Ouchterlony analysis. It did not detect von Willebrand's disease antigen II (22). In the radio crossed immunoelectrophoresis studies 50- $\mu$ l samples were used with VIIIR:Ag concentrations varying from 0.025 to $0.15 \mathrm{U} / \mathrm{ml}$. Freeze-thawing or Triton X-100 treatment of plasma VIIIR:Ag did not affect its appearance on radio-crossed immunoelectrophoresis or its quantitation by radio quantitative immunoelectrophoresis.

VIIIR:C was measured by a partial thromboplastin time technique (28) and platelet-ristocetin cofactor activity (VIIIR: RCo) by a tilt tube technique with fixed platelets (29).

The lysosomal enzymes, $\beta$-glucuronidase and $N$-acetyl- $\beta$ glucosaminidase were assayed in the supernate of aggregated platelets by techniques described elsewhere $(30,31)$. Lactic dehydrogenase (LDH) was measured in the supernate to detect cytoplasmic leakage as a result of platelet lysis. The assay was performed by a DuPont ACA (Automatic Chemical Analyzer), DuPont Instruments, Wilmington, Del., according to the manufacturer's instruction. 5HT release was measured by adding $0.03 \mathrm{ml}$ supernate to $10 \mathrm{ml}$ Aquasol (New England Nuclear) and counting the radioactivity in a liquid scintillation counter (Beckman LS 3133P; Beckman Instruments, Inc., Palo Alto, Calif.).

Fibrinogen quantitation. Fibrinogen released from the platelet was determined by a radioimmunoasay of the double antibody type as described $(32,33)$.

von Willebrand's disease with atypical VIIIR:Ag. Platelets isolated from a patient with von Willebrand's disease whose plasma VIIIR:Ag lacked the less anodal (slower) migrating forms on crossed immunoelectrophoresis, were aggregated with ADP, $0.1 \mathrm{mM}$; thrombin, $0.5 \mathrm{U} / \mathrm{ml}$; and collagen, 100 $\mu \mathrm{g} / \mathrm{ml}$. This patient had a lifelong history of excessive bleeding and had a skin bleeding time of $>20 \mathrm{~min}$ (normal $=<8.5$ min). The plasma level of VIIIR:Ag was $0.25 \mathrm{U} / \mathrm{ml}$, VIIIR:C was $0.37 \mathrm{U} / \mathrm{ml}$, and VIIIR:RCo was $0.15 \mathrm{U} / \mathrm{ml}$.

\section{RESULTS}

Platelets washed by albumin density gradient and gel filtration were obtained in a final concentration of between 5.5 and $8 \times 10^{8}$ platelets $/ \mathrm{ml}$. Contaminating VIIIR:Ag in the supernate was $<0.0005 \mathrm{U} / \mathrm{ml}$ and none was detectable throughout the course of the experiments unless stimulating agents were added. The washed platelets did not aggregate with ristocetin in the absence of added VIIIR:Ag. Total platelet VIIIR:Ag was determined by measuring the VIIIR:Ag released into the supernate after lysis of the platelets with $1 \%$ Triton X-100 for $1 \mathrm{~h}$ at $37^{\circ} \mathrm{C}$. Eight platelet preparations from different donors gave a total platelet VIIIR:Ag content of $0.17 \mathrm{U}(\mathrm{SE} \pm 0.01) / 5 \times 10^{8}$ platelets. Triton $\mathrm{X}-100$ lysis of five separate preparations of washed platelets from the same donor gave a total platelet VIIIR:Ag content of $0.15 \mathrm{U}(\mathrm{SE} \pm 0.004) / 5 \times 10^{8}$ platelets. Lysis of platelets with the freeze-thaw technique gave an average total platelet content $10 \%$ less than obtained with Triton X-100.

Collagen studies. Significant release of VIIIR:Ag was detected at a concentration of collagen of $5 \mu \mathrm{g} /$ $\mathrm{ml}$ and maximum release was always obtained with $50 \mu \mathrm{g}$ (Fig. 1). Maximum release of VIIIR:Ag occurred at significantly lower concentrations of collagen than was necessary for maximum release of $5 \mathrm{HT}$ and the lysosomal enzymes. The maximum release of each component studied at $10 \mathrm{~min}$ after collagen stimulation, as a percentage of the total platelet content of each was: VIIIR:Ag, $26 \%$ (SE \pm 3 ); $5 \mathrm{HT}, 80 \%$ (SE \pm 5$)$; $\mathrm{N}$ acetyl- $\beta$-D-glucosaminidase, $32 \%(\mathrm{SE} \pm 3)$; $\beta$-glucuronidase, $22 \%(\mathrm{SE} \pm 2)$. No significant $\mathrm{LDH}$ was released during the course of any study reported here. In the 60-min incubation studies, no additional significant 5HT or lysosomal enzyme release was detected, but the maximum amount of VIIIR:Ag released increased to $50 \%(\mathrm{SE} \pm 6)$ of the total platelet content. However; the concentration of collagen required to give maximum VIIIR:Ag release did not differ significantly between the 10- and 60-min studies (Fig. 2).

The time-course of VIIIR:Ag release was studied over a period of $1 \mathrm{~h}$ with collagen at concentrations of $50 \mu \mathrm{g} / \mathrm{ml}$ (Fig. 3). Almost $50 \%$ of the release of VIIIR:Ag was accomplished within $5 \mathrm{~min}$. The release of $5 \mathrm{HT}$ and $N$-acetyl- $\beta$-D-glucosaminidase (the latter not shown in Fig. 3) was even more rapid; both reaching an apparent maximum within 5 min. However, both 5HT and lysosomal enzyme release also appeared to show some progressive diminution of release rate with time, but the rate was so rapid that it was difficult to define the course with the same accuracy and clarity as could be obtained with VIIIR:Ag release.

In combination, antimycin A and 2-deoxy-D-glucosereduced collagen-induced release of VIIIR:Ag by $90 \%$

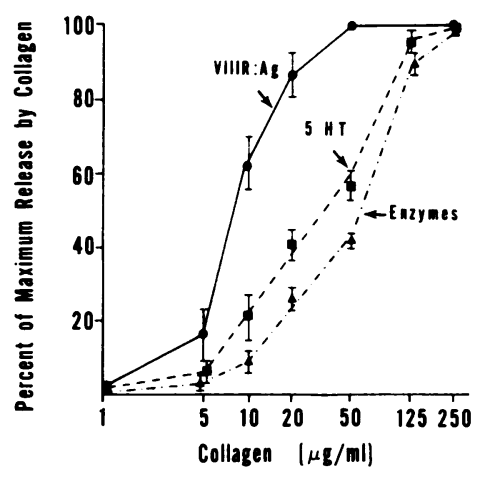

Figure 1 Dose-response curve of collagen-induced release. Final collagen concentration is plotted on the horizontal, logarithmic axis. The means and standard errors of released VIIIR:Ag, 5HT and $N$-acetyl- $\beta$-D-glucosaminidase (enzymes) from five $10-\mathrm{min}$ incubation studies, expressed as a percentage of the maximum amount of each released at any collagen concentration, are plotted on the vertical, arithmetic axis. 


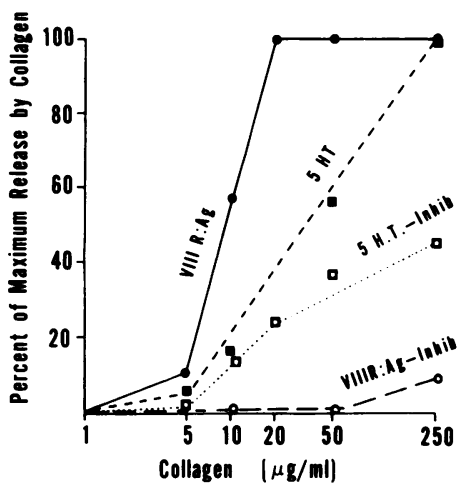

FIGURE 2 Inhibition of collagen-induced release by metabolic inhibitors (Inhib). Two experiments were performed in which washed platelets were incubated for $30 \mathrm{~min}$ at $37^{\circ} \mathrm{C}$ with and without antimycin $A$ and 2-deoxy-D-glucose. VIIIR:Ag and 5HT released into the supernate were assayed $1 \mathrm{~h}$ after the addition of collagen at varying concentrations. All parameters are plotted as in Fig. 1 except that each point represents the mean of two values.

or more. Lysosomal enzyme release was completely inhibited and $5 \mathrm{HT}$ release was reduced to $\cong 50 \%$ of the normal response (Figs. 2 and 3).

Thrombin studies. Significant release of VIIIR:Ag was regularly detected at $0.025 \mathrm{U} / \mathrm{ml}$ thrombin and maximum release was always obtained at $0.5 \mathrm{U} / \mathrm{ml}$ thrombin. The dose-response curve of VIIIR:Ag release in response to thrombin was not significantly different to that of 5HT release, but both were different from the thrombin-induced dose response of lysosomal enzyme release from the platelets (Fig. 4). Maximum release of VIIIR:Ag after incubation periods of 10 and 60 min was achieved at the same thrombin concentration.

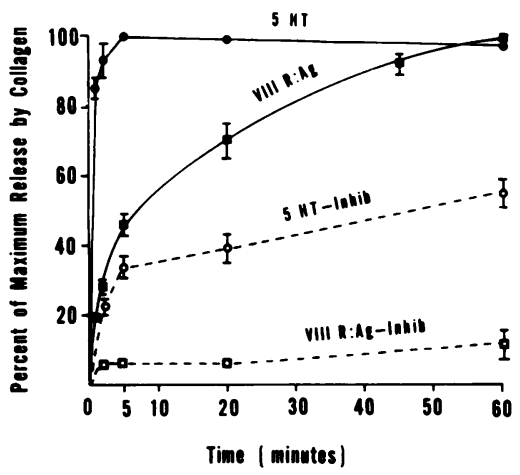

FIgURE 3 Time-course of collagen-induced release of VIIIR: Ag and 5HT and the effect of metabolic inhibitors (Inhib). Five experiments were performed, including three in which washed platelets were incubated at $37^{\circ} \mathrm{C}$ for $30 \mathrm{~min}$ with and without added antimycin $A(4 \mu \mathrm{g} / \mathrm{ml}$, final concentration) and 2-deoxy-D-glucose (6 mM). Aggregation was induced with $50 \mu \mathrm{g}$ final concentration of collagen and the release reaction was stopped at specific time intervals thereafter by the addition of formalin-EDTA. VIIIR:Ag and 5HT are plotted as in Fig. 1.

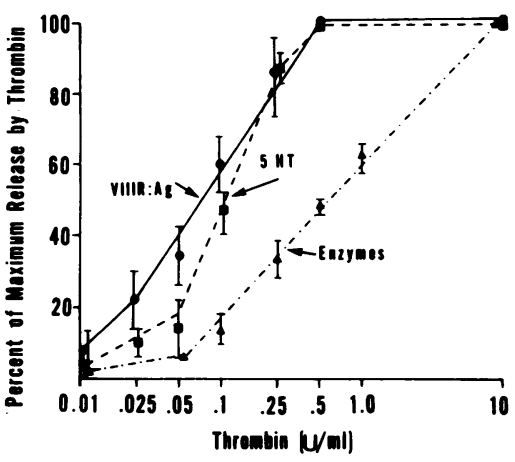

FIGURE 4 Dose-response curve of thrombin-induced release. Five experiments, terminated $10 \mathrm{~min}$ after the addition of thrombin, were performed. All parameters are plotted as in Fig. 1.

Thus, although the absolute amount of VIIIR:Ag increased between these two time points, it did so proportionately at all thrombin concentrations. The maximum amount of VIIIR:Ag released by thrombin (33\% of the total platelet VIIIR:Ag) was lower than for collagen, although more $5 \mathrm{HT}$ and $N$-acetyl- $\beta$-glucosaminidase were released by thrombin (Table I). In seven experiments, in which both collagen- and thrombininduced release were studied simultaneously over an incubation period of $1 \mathrm{~h}$, the thrombin-induced maximum release was $59 \%(\mathrm{SE} \pm 6)$ of the maximum VIIIR:Ag released by collagen.

Time-course studies of release of 5HT and VIIIR:Ag by thrombin $(0.5 \mathrm{U} / \mathrm{ml})$ gave essentially identical results to those obtained with collagen (Fig. 5). Release was most rapid initially with almost $50 \%$ of the release of VIIIR:Ag being accomplished within 5 min.

The metabolic inhibitors, antimycin A and 2-deoxyD-glucose, significantly inhibited thrombin-induced 5HT, lysosomal enzyme and VIIIR:Ag release. As with

TABLE I

ADP-Induced Release of VIIIR:Ag and 5HT

\begin{tabular}{ccc}
\hline Final ADP concentration & $\begin{array}{c}\text { VIIIR:Ag } \\
\text { release }\end{array}$ & 5HT release \\
\hline & & $\%$ \\
$0.1 \mathrm{mM}$ & $22(\mathrm{SE} \pm 4)$ & $21(\mathrm{SE} \pm 2)$ \\
$50 \mu \mathrm{M}$ & $19(\mathrm{SE} \pm 5)$ & $17(\mathrm{SE} \pm 3)$ \\
$10 \mu \mathrm{M}$ & $16(\mathrm{SE} \pm 5)$ & $14(\mathrm{SE} \pm 3)$ \\
$3 \mu \mathrm{M}$ & $8(\mathrm{SE} \pm 3)$ & $10(\mathrm{SE} \pm 2)$ \\
$1 \mu \mathrm{M}$ & 0 & 2 \\
$3 \mu \mathrm{M}$ & 0 & 0
\end{tabular}

Total platelet VIIIR:Ag: $0.15 \mathrm{U}(\mathrm{SE} \pm 0.008) / 5 \times 10^{8}$ platelets. ADP-induced release of VIIIR:Ag and 5HT over an incubation period of $1 \mathrm{~h}$. VIIIR:Ag and 5HT released at varying concentrations of ADP from five experiments are expressed as a mean and standard error of the total platelet content of each. 


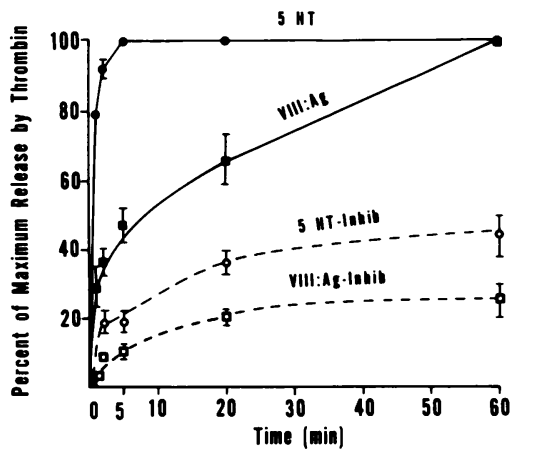

FIgURE 5 Time-course of thrombin-induced release of VIIIR:Ag and 5HT and the effect of metabolic inhibition (Inhib). Five experiments with noninhibited platelets and three with inhibitors, as described in Fig. 2 were performed.

collagen-induced release, VIIIR:Ag release was more markedly inhibited than 5HT release at these concentrations of inhibitors (Fig. 5). No LDH was released at any concentration of thrombin with or without inhibitors.

ADP studies. The response to ADP of washed platelets proved to be more variable than the response to collagen or thrombin. Platelets which had been washed and gel filtered showed a poor release response $(<15 \%$ total $5 \mathrm{HT}$ ) at all concentrations of ADP. By omitting the gel filtration step, $>20 \%$ release of total $5 \mathrm{HT}$ could usually be achieved at a maximum ADP concentration of between $10 \mu \mathrm{M}$ and $0.1 \mathrm{mM}$. Wherever significant 5HT release occurred, VIIIR:Ag was also released. At a concentration of $1 \mu \mathrm{M}$ ADP, no significant release of 5HT or VIIIR:Ag was obtained in five experiments. At concentrations of between $10 \mu \mathrm{M}$ and $0.1 \mathrm{mM}$ between 20 and $30 \%$ release of total platelet $5 \mathrm{HT}$ and VIIIR:Ag occurred. Table I summarizes the results of five experiments.

With any one experiment, however, the release response at a given concentration was consistent, so that time-course studies were possible. In five such studies performed at a final ADP concentration of $50 \mu \mathrm{M}$, the time-course results were not significantly different from those obtained with collagen and thrombin. Release of VIIIR:Ag reached an apparent plateau after $60 \mathrm{~min}$, but $42 \%(\mathrm{SE} \pm 4 \%)$ of this release had occurred within 5 min of ADP stimulation. No LDH was released by any concentration of ADP.

Comparative time-courses of collagen-induced VIIIR: Ag and fibrinogen release. Triton X-100 lysed platelets were found to release a total of $40 \mu \mathrm{g}$ of fibrinogen $/ 5$ $\times 10^{8}$ platelets. $1 \mathrm{~h}$ after stimulation with collagen $(100$ $\mu \mathrm{g} / \mathrm{ml}$, final concentration) $58 \%$ (SE \pm 11 ) of this plateletcontained fibrinogen was released. The metabolic inhibitors, antimycin A and 2-deoxy-D-glucose, inhibited 90\% of this release. In five experiments, $59 \%(\mathrm{SE} \pm 6.5)$ of fibrinogen release had occurred within 5 min of col- lagen stimulation and $82 \%(\mathrm{SE} \pm 8)$ within $20 \mathrm{~min}$. Of the VIIIR:Ag released in these experiments, $38 \%$ $(\mathrm{SE} \pm 3)$ had occurred within $5 \mathrm{~min}$ and $63 \%(\mathrm{SE} \pm 8)$ within $20 \mathrm{~min}$.

VIIIR:C and VIIIR:WF. No significant VIIIR:C or VIIIR:RCo activity was detected in association with VIIIR:Ag released from platelets. However, VIIIR:Ag levels obtained were always $<0.2 \mathrm{U} / \mathrm{ml}$ and the existing techniques are not sensitive enough, in our hands, to detect corresponding levels of VIII:RCo with confidence.

Crossed immunoelectrophoresis analyses of platelet VIIIR:AG. Crossed immunoelectrophoresis showed consistent differences between the VIIIR:Ag released by collagen and ADP as compared to thrombin (Fig. 6). Collagen and ADP-released VIIIR:Ag had electrophoretic characteristics similar to plasma VIIIR:Ag. Thrombin-released VIIIR:Ag, on the other hand, was depleted of the less anodal (slower) migrating forms with only the more anodal (faster) migrating forms found in plasma being present.

There was also less obvious, but consistent, differences between the VIIIR:Ag obtained by Triton X-100 and freeze-thaw lysis and plasma. Triton X-100 lysed platelets released relatively more of the slower migrating forms of VIIIR:Ag as compared to plasma, whereas freeze-thaw lysed platelets released VIIIR:Ag which was relatively depleted of these forms as compared to plasma (or collagen and ADP-released VIIIR:Ag). The addition of thrombin to collagen-released VIIIR:Ag, or of highly purified VIIIR:Ag to the supernate of thrombin-stimulated platelets and freeze-thaw lysed platelets, caused no detectable quantitative or qualitative alteration of VIIIR:Ag.

Platelet VIIIR:Ag in von Willebrand's disease. The platelets from a patient with von Willebrand's disease whose plasma lacked the slower migrating forms of VIIIR:Ag were found to contain 0.13 U VIIIR:Ag/5 $\times 10^{8}$ platelets. The patient's platelets also released VIIIR:Ag normally: 40\% of total platelet VIIIR:Ag being released by collagen, $25 \%$ by thrombin, and $30 \%$ by ADP. The collagen and ADP-released VIIIR:Ag had the same electrophoretic characteristics as the VIIIR:Ag in the patient's plasma (Fig. 7). Thus, compared to the VIIIR:Ag released by collagen and ADP from normal platelets, the slower migrating forms of VIIIR:Ag were depleted in this patient. The VIIIR:Ag released by thrombin was electrophoretically different from the patient's plasma VIIIR:Ag in that there was a still further depletion of slower migrating VIIIR:Ag forms (Fig. 7). Thrombin-released VIIIR:Ag from normal platelets was also depleted of the slower migrating forms and did not differ from the patient's thrombin-released VIIIR:Ag. Ruggeri et al. (34) have reported that the VIIIR:Ag released from platelets of similar von Willebrand's patients by hypotonic glycerol lysis showed a faster elec- 


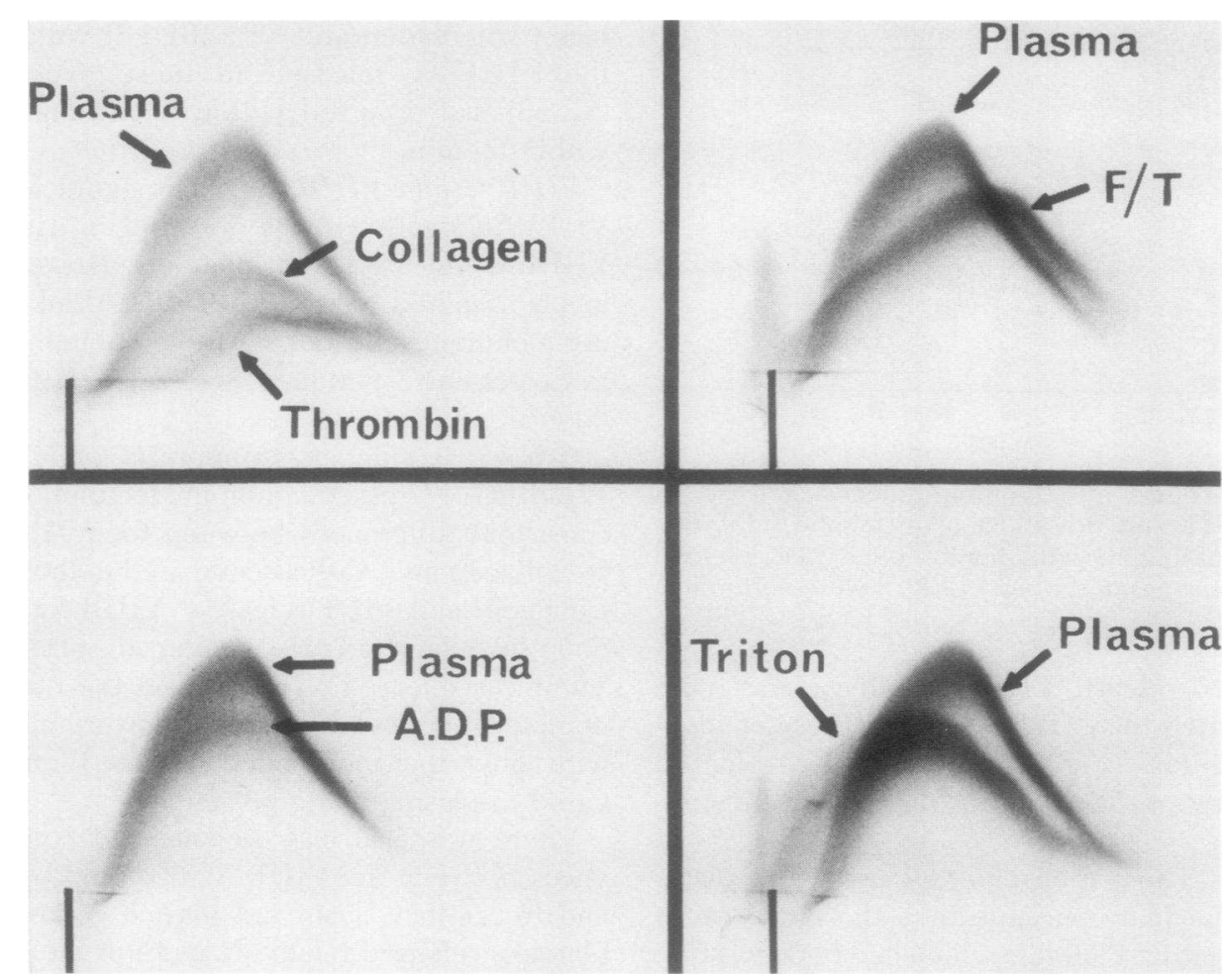

FIGURE 6 Radio-crossed immunoelectrophoresis autoradiograph patterns of VIIIR:Ag released from normal platelets $10 \mathrm{~min}$ after stimulation by thrombin $(0.5 \mathrm{U} / \mathrm{ml})$, collagen $(50 \mu \mathrm{g} / \mathrm{ml}), \mathrm{ADP}(50$ $\mu \mathrm{M})$, or after repeated freeze-thawing $(\mathrm{F} / \mathrm{T})$ or Triton X-100 (Triton) treatment. Autoradiograph platelet VIIIR:Ag patterns have been superimposed on the pattern obtained from the plasma VIIIR:Ag of the same person. The sample origin is indicated by the vertical bar in the bottom lefthand corner of each radio-crossed immunoelectrophoresis. The anode is to the right in the first dimension and to the top in the second dimension.

trophoretic mobility as compared to that released from normal platelets. Our results, with different methods of lysis, confirmed this observation and also provided additional observations. Freeze-thaw lysis of our patient's platelets released VIIIR:Ag significantly different from that released by freeze-thaw lysis of normal platelets. However, freeze-thaw lysis of normal platelets resulted in the release of VIIIR:Ag which was depleted of the slower migrating forms compared to normal plasma (Fig. 6). Freeze-thaw lysis of the patient's platelets, on the other hand, released VIIIR:Ag that was electrophoretically identical to her plasma VIIIR:Ag (Fig. 7). In addition, we found that detergent lysis with Triton X-100 of the patient's platelets resulted in the release of slow migrating forms of VIIIR:Ag that were not present in her plasma (Fig. 7). However, a similar phenomenon occurred with Triton X-100 lysis of normal platelets (Fig. 6) and the net effect was that the patient's Triton X-100-released VIIIR:Ag still lacked some of the slower migrating forms present in normal platelets.

\section{DISCUSSION}

A significant proportion of platelet-contained VIIIR:Ag appears to exist in a secretory pool which can be mobilized to varying degrees by ADP, collagen, and thrombin. Release of VIIIR:Ag by these agents is dependent on active metabolic processes within the platelets because it is markedly depressed by agents that inhibit the platelet's energy metabolism. This would indicate that VIIIR:Ag release is a true secretory reaction and not the result of some form of proteolytic digestion of platelet membrane-bound VIIIR:Ag. In support of this contention is the fact that, using the highly sensitive two-dimensional technique of radioimmunoelectrophoresis, no forms of released VIIIR:Ag could be detected with migration other than that found normally in plasma.

The method we used to wash platelets renders them relatively insensitive to ADP (35) and the concentrations of ADP necessary to induce serotonin and VIIIR:Ag release were relatively high. This method of 


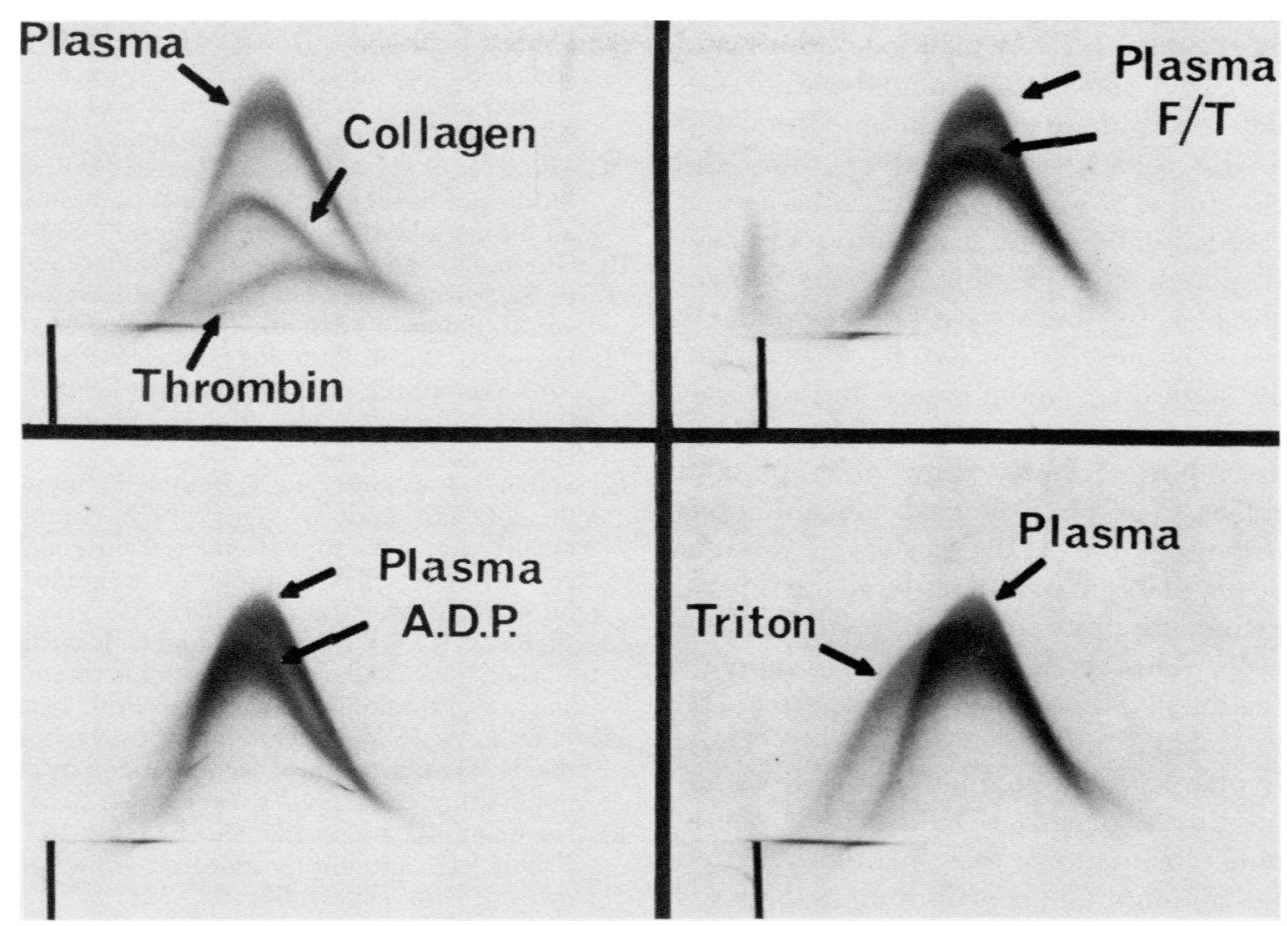

FIGURE 7 Radio-crossed immunoelectrophoresis autoradiograph patterns of VIIIR:Ag released from platelets of a patient with von Willebrand's disease is compared to the plasma VIIIR:Ag of that patient by superimposition as in Fig. 6. The patterns obtained for plasma VIIIR:Ag and for ADPreleased or freeze-thaw lysis-released platelet VIIIR:Ag overlap most completely and are difficult to differentiate.

washing was used to assure that the platelets were free of external VIIIR:Ag (36). However, the concentrations of ADP necessary for VIII:Ag release were no greater than those necessary for serotonin release.

Although the data presented in this report does not give definitive information as to the nature of the VIIIR:Ag pool, the differences in the dose-response dynamics between VIIIR:Ag and both 5HT and lysosomal enzyme release and the differential effects of the metabolic inhibitors raises the possibility that VIIIR:Ag resides in a different pool from the dense granules and lysosomal enzyme-containing $\alpha$-granules of the platelet.

The time-course pattern of VIIIR:Ag release is consistent with a single stimulatory event that induces a rapid initial release. As might be anticipated, the rate of these events progressively diminishes with time as a result of the depletion of available releasable material. The active metabolic processes initiated in platelets by ADP, collagen, or thrombin are thought to lead to the movement of storage granules to the platelet membrane and the fusion of the two (37). Subsequent events, such as the movement of substances out of the granules and through the platelet canalicular system to the outside, are influenced by the diffusion constant of the molecules as well as possible interactions with the platelet surface. Diffusion constants of molecules are inversely proportional to size and it is not unexpected that VIIIR: $\mathrm{Ag}$, with a molecular weight of up to $5 \times 10^{6}$ daltons, diffuses significantly more slowly than $5 \mathrm{HT}$ or the lysosomal enzymes. There was a significant increase, with time, of the proportion of larger, more slowly migrating forms of VIIIR:Ag released. This suggests that molecular size and rate of release are correlated. Similarly, fibrinogen $(340,000 \mathrm{~mol} \mathrm{wt})$ has a rate of release intermediate to VIIIR:Ag, on the one hand, and the lysosomal enzymes and 5HT on the other.

The electrophoretic characteristics of plateletderived VIIIR:Ag differed according to the techniques used to release it. ADP and collagen released VIIIR-Ag from platelets that were identical to the VIIIR:Ag in plasma. Thrombin-released VIIIR:Ag, however, was depleted of the slower migrating forms. Freeze-thaw lysis also led to the release of VIIIR:Ag which was relatively depleted of the slower migrating forms, whereas lysis by the detergent Triton X-100 released proportionately more of these forms as compared to normal plasma.

These differences cannot be readily attributed to proteolysis. Purified VIIIR:Ag or collagen-released platelet VIIIR:Ag was not altered in its electrophoretic mobility by incubation with thrombin or collagenreleased platelet products, or material released from 
platelets by freeze-thaw lysis of platelets. Koutts and Zimmerman (38), have shown that isolated membranes from lysed platelets and intact, thrombin-stimulated platelets have the capacity to selectively bind the slower migrating forms of VIIIR:Ag. It is probable that VIIIR:Ag released after thrombin stimulation or freezethaw lysis of platelets is depleted of the slower migrating forms because these have been selectively bound to the membranes. Triton X-100, on the other hand, partially solubilizes membranes and releases slowly migrating, membrane-bound VIIIR/Ag (38).

The results reported here show that platelet VIIIR:Ag is released by physiological concentrations of collagen, thrombin, and ADP. At sites of vascular damage, where platelets amass and undergo the release reaction, high concentrations of VIIIR:Ag will be made locally available. This platelet VIIIR:Ag supports platelet aggregation in the presence of ristocetin $(6,23)$ and binds to isolated platelet membranes. ${ }^{2}$ Thus, with respect to platelet function, platelet VIIIR:Ag has the same properties as plasma VIIIR:Ag. It is, therefore, reasonable to assume that platelet-released VIIIR:Ag makes an important contribution to the function of platelets during the primary hemostatic process.

\section{ACKNOWLEDGMENTS}

The authors wish to thank Jim Roberts, Kimie Hirabayashi, and Janet Plescia for their skillful technical assistance and Sharon Dinwiddie for preparing the manuscript.

This work was supported by National Institutes of Health grants HL-15491, HL-16411, 14217, and 20517. This is publication no. 1519 from Scripps Clinic and Research Foundation.

\section{REFERENCES}

1. Zimmerman, T. S., O. D. Ratnoff, and A. E. Powell. 1971. Immunological differentiation of classic hemophilia (Factor VIII deficiency) and von Willebrand's disease. J. Clin. Invest. 50: 244-254.

2. Peake, I. R., A. L. Bloom, and J. C. Giddings. 1974. Inherited variants of Factor VIII-related proteins in von Willebrand's disease. N. Engl. J. Med. 291: 113-117.

3. Howard, M. A., R. J. Sawers, and B. G. Firkin. 1973. Ristocetin: a means of differentiating von Willebrand's disease into two groups. Blood. 41: 687-690.

4. Tchopp, T. B., H. J. Weiss, and H. R. Baumgartner. 1974. Decreased adhesion of platelets to subendothelium in von Willebrand's disease.J. Lab. Clin. Med. 83: 296-300.

5. Howard, M. A., D. C. Montgomery, and R. M. Hardisty. 1974. Factor VIII-related antigen in platelets. Thromb. Res. 4: 617-624.

6. Nachman, R. L., and E. A. Jaffe. 1975. Subcellular platelet Factor VIII antigen and von Willebrand Factor. J. Exp. Med. 141: 1101-1112.

7. Andersen, J, and H. Gralnick, and L. Corash. 1976. Partial characterization of platelet-associated Factor VIII. Circulation. 53(Suppl. II): 116. (Abstr.)

\footnotetext{
${ }^{2}$ Koutts, J. and T. S. Zimmerman. Submitted for publication.
}

8. Bouma, B. N., J. M. Hordijk-Hos, S. deGraaf, J. J. Sixma, and J. A. van Mourik. 1975. Presence of Factor VIIIrelated antigen in blood platelets of patients with von Willebrand's disease. Nature (Lond.). 257: 510-512.

9. Coller, B. S., R. J. Hirschman, and H. R. Gralnick. 1975. Studies of the Factor VIII/von Willebrand factor antigen on human platelets. Thromb. Res. 6: 469-480.

10. Green, D., and E. V. Potter. 1976. Platelet-bound ristocetin aggregation factor in normal subjects and patients with von Willebrand's disease.J. Lab. Clin. Med. 87: 976-986.

11. Hoyer, L. W., R. P. de los Santos, and J. R. Hoyer. 1973. Anti-hemophilic Factor antigen: localization in endothelial cells by immunofluorescent microscopy. J. Clin. Invest. 52: 2737-2744.

12. Sixma, J. J., L. Kater, B. N. Bouma, F. Schmitz du Moulin, S. deGraaf, and G. Tuit. 1976. Immunofluorescent localization of Factor VIII-related antigen, fibrinogen and several other plasma proteins in hemostatic plugs in humans. J. Lab. Clin. Med. 87: 112-119.

13. Bloom, A. L., J. C. Giddings, and C. J. Willis. 1973. Factor VIII on the vascular intima: possible importance in hemostasis and thrombosis. Nat. New Biol. 241: 217-219.

14. Jaffe, E. A., L. W. Hoyer, and R. L. Nachman. 1973. Synthesis of antihemophilic factor antigen by cultured human endothelial cells. J. Clin. Invest. 52: 2757-2764.

15. Nachman, R., R. Levine, and E. Jaffe. 1977. Synthesis of Factor VIII antigen by cultured guinea pig megakaryocytes. J. Clin. Invest. 60: 911-921.

16. Walsh, P. N., D. C. B. Mills, and J. G. White. 1977. Metabolism and function of human platelets washed by albumin density gradient separation. Br.J. Haematol. 36: 281-296.

17. Holmsen, H., C. A. Setkowsky, and J. H. Day. 1974. Effects of antimycin and 2-deoxyglucose on adenine nucleotides in human platelets. Biochem. J. 144: 385-396.

18. Wilner, G. D., H. L. Nossel, and E. C. LeRoy. 1968. Activation of Hageman Factor by collagen. J. Clin. Invest. 47: 2608-2615.

19. Wilner, G. D., H. L. Nossel, and E. C. LeRoy. 1968. Aggregation of platelets by collagen. J. Clin. Invest. 47: 2616-2621.

20. Fenton, J. W., II, M. J. Fasco, A. B. Stackrow, D. L. Aronson, A. M. Young, and J. S. Finlayson. 1977. Human Thrombin. Production, evaluation and properties of $\alpha$ thrombin. J. Biol. Chem. 252: 3587-3598.

21. Holmsen, H., and C. A. Setkowsky-Dangelmaier. 1977. Adenine nucleotide metabolism of blood platelets. Biochim. Biophys. Acta. 497: 46-61.

22. Montgomery, R. R., and T. S. Zimmerman. 1978. von Willebrand's disease antigen II. A new plasma and platelet antigen deficient in severe von Willebrand's disease. J. Clin. Invest. 61: 1498-1507.

23. Zimmerman, T. S., J. Roberts, and T. S. Edgington. 1975. Factor VIII-related antigen: multiple molecular forms in human plasma. Proc. Natl. Acad. Sci. U. S. A. 72: 51215125.

24. Zimmerman, T. S., L. W. Hoyer, L. Dickson, and T. S. Edgington. 1975. Determination of the von Willebrand's disease antigen (Factor VIII-related antigen) in plasma by quantitative immunoelectrophoresis. J. Lab. Clin. Med. 86: $152-159$.

25. Ternyck, T., and S. Avrameas. 1976. Polyacrylamideprotein immunoadsorbents prepared with glutaraldehyde. FEBS (Fed. Eur. Biochem. Soc.) Lett. 23: 24-28.

26. Louvard, E., S. Maroux, and P. Desnuelle. 1975. Topological studies on the hydrolases bound to the intestinal brush 
border membrane. II. Interaction of free and bound aminopeptidase with a specific antibody. Biochim. Biophys. Acta. 389: 389-400.

27. McConahey, P. J., and F. J. Dixon. 1966. A method of trace iodination of proteins for immunologic studies. Int. Arch. Allergy Appl. Immunol. 29: 185-189.

28. Simone, J. V., J. Vanderheiden, and C. F. Abildgaard. 1967. A semiautomatic one-stage Factor VIII assay with a commercially prepared standard. J. Lab. Clin. Med. 69: 706-712.

29. Allain, J. P., H. A. Cooper, R. H. Wagner, and K. M. Brinkhous. 1975. Platelets fixed with paraformaldehyde: a new reagent for assay of von Willebrand Factor and platelet aggregating factor.J. Lab. Clin. Med. 85: 318-328.

30. Fishman, W. H., B. Springer, and R. Brunetti. 1948. Application of an improved glucuronidase assay method to the study of human blood $\beta$-glucuronidase. J. Biol. Chem. 173: 449-456.

31. Woollen, J. W., R. Heyworth, and P. G. Walker. 1961. Studies in glucosaminidase. 3. Testicular $\mathrm{N}$-acetyl- $\beta$ glucosaminidase and $\mathrm{N}$-acetyl- $\beta$-galactosaminidase. $\mathrm{BiO}$ chem. J. 78: 111-116.
32. Plow, E. F., and T. S. Edgington. 1973. Immunobiology of fibrinogen. J. Clin. Invest. 52: 273-282.

33. Plow, E. F., and T. S. Edgington, 1975. Unique immunochemical features of intracellular stability of platelet fibrinogen. Thromb. Res. 7: 729-742.

34. Ruggeri, Z. M., P. M. Manucci, R. Baeler, and T. Barbui. 1978. Factor VIII-related properties in platelets from patients with von Willebrand's disease. J. Lab. Clin. Med. 91: $132-140$.

35. Sixma, J. J. 1976. Platelet Isolation. In Platelet Function Testing. H. J. Day, H. Holmsen, and M. B. Zucker, editors. Proceedings of a Conference on Platelet Function Testing. Department of Health, Education, and Welfare no. (NIH) 78-1087. 34-46.

36. Timmons, S., and J. Hawiger. 1978. Separation of human platelets from plasma proteins including Factor VIII VwF $_{\text {F }}$ by a combined albumin gradient-gel filtration method using hepes buffer. Thromb. Res. 12: 297-306.

37. White, J. G. 1974. Electron microscopic studies of platelet secretion. In Progress in Hemostasis and Thrombosis. T. H. Spaet, editor. Grune \& Stratton, Inc., New York. 2: 93. 